\title{
Dedifferentiated fat cells: an alternative source of adult multipotent cells from the adipose tissues
}

\author{
Jie-fei Shen ${ }^{1,2}$, Atsunori Sugawara ${ }^{1}$, Joe Yamashita $^{1}$, Hideo $_{\text {Ogura }}{ }^{2}$, Soh Sato $^{1 *}$ \\ ${ }^{1}$ Department of Periodontology, School of Life Dentistry at Niigata, Nippon Dental University, Niigata 951-1500, \\ Japan; ${ }^{2}$ Department of Dental Materials, School of Life Dentistry at Niigata, Nippon Dental University, Niigata \\ 951-1500, Japan
}

When adipose-derived stem cells (ASCs) are retrieved from the stromal vascular portion of adipose tissue, a large amount of mature adipocytes are often discarded. However, by modified ceiling culture technique based on their buoyancy, mature adipocytes can be easily isolated from the adipose cell suspension and dedifferentiated into lipid-free fibroblast-like cells, named dedifferentiated fat (DFAT) cells. DFAT cells re-establish active proliferation ability and undertake multipotent capacities. Compared with ASCs and other adult stem cells, DFAT cells showed unique advantages in their abundance, isolation and homogeneity. In this concise review, the establishment and culture methods of DFAT cells are introduced and the current profiles of their cellular nature are summarized. Under proper induction culture in vitro or environment in vivo, DFAT cells could demonstrate adipogenic, osteogenic, chondrogenic and myogenic potentials. In angiogenic conditions, DFAT cells could exhibit perivascular characteristics and elicit neovascularization. Our preliminary findings also suggested the pericyte phenotype underlying such cell lineage, which supported a novel interpretation about the common origin of mesenchymal stem cells and tissue-specific stem cells within blood vessel walls. Current research on DFAT cells indicated that this alternative source of adult multipotent cells has great potential in tissue engineering and regenerative medicine.

Keywords: dedifferentiated fat cells; adult stem cells; adipose tissue; mesenchymal stem cells; regenerative medicine International Journal of Oral Science (2011) 3: 117-124. doi: 10.4248/IJOS 11044

Adipose tissue, because of its abundance and relatively less invasive harvest methods, represents a practical and appealing source of mesenchymal stem cells (MSCs) [1]. It has been demonstrated by many groups that MSCs within the stromal-vascular fraction (SVF) of subcutaneous adipose tissue display multilineage plasticity in vitro and in vivo [2-6]. Several terms have been used to name these pluripotent adult progenitor cells, such as adipocyte precursor cells [7], preadipocytes [8], adipose-

*Correspondence: Soh Sato Tel: 0081252671500 ext. 337; Fax: 0081252333983

E-mail: s-sato@ngt.ndu.ac.jp

Received 10 March 2011; Accepted 17 May 2011 derived adult stem (ADAS) cells [9], adipose-derived stromal cells [10], adipose-derived adherent stromal cells (ADASC) [1], processed lipoaspirate cells [11], and adipose-derived stem cells (ASCs). According to the consensus reached in the 2004 IFATS (Annual International Fat Applied Technology Society) meeting, more researchers have currently adopted the term, ASCs [12]. Common method to obtain ASCs is to isolate the SVF from the adipose tissue by mechanical dissociation and enzymatic treatment, followed by fluorescence activated cell sorting (FACS) or culture selection [6]. However, because the adipose SVF portion is composed of heterogeneous groups of cells and there is short of specific markers for ASCs, it remained a challenge to 
isolate purified ASCs and proceed with further bioengineering applications [12].

The adipocytes, other than the cell groups within the SVF portion, can be dedifferentiated into fibroblast-like cells named the dedifferentiated fat (DFAT) cells by ceiling culture strategy based on their buoyant property [13-15]. With this method and provided the fact that the mature adipocytes are the most abundant cell type in the adipose tissue, the DFAT cells which exhibit proliferation and multipotent capacity in vitro and in vivo have been regarded as an ideal source for adult postnatal pluripotent cells of much higher homogeneity than ASCs $[14,16-19]$. In this review, by a briefing summary on the origin, identification and differentiation ability of DFAT cells, we're going to unveil the great application potentials of this unique cell kind in tissue engineering and regenerative medicine.

\section{Establishment of DFAT cells}

At beginning, the source of DFAT cells was the adipose tissue from the bone marrow of metacarpal bones in neonatal calves [13, 20]. Following studies proved that DFAT cells can also be derived from the subcutaneous white adipose tissue (WAT) from various species, including human [17], rats [16], mouse [18] and cattle [8]. Because of the wide application of free-fat transfer and plastic liposuction [21-22], large amount of human DFAT cells can be derived by those convenient ways for autologous tissue engineering and reconstruction purposes. The dispersion and digestion process of WAT resembles the routine method of ASCs culture. Then, the following dedifferentiation method to obtain DFAT cells commonly known as ceiling culture technique, was first reported by Sugihara at 1986 [13]. Unlike the SVF in the mixed solution of digested fat tissue, which sinks to the bottom, the mature adipocytes will float to the liquid surface. In a filled culture flask, the buoyant adipocytes will attach to the inner side of the roof. The adhered unilocular adipocytes will be flattened losing a round contour and the dominant lipid portion will break into several smaller droplets to exhibit a multiocular outlook (Figure 1). According to the different studies $[14,16-17,19,23]$, in the following 2 to 3 weeks of uninterrupted culture, the fat drops within the adipocytes will gradually disappear and the cells shift into fibroblast-like morphology, reestablishing a strong proliferation ability as well.

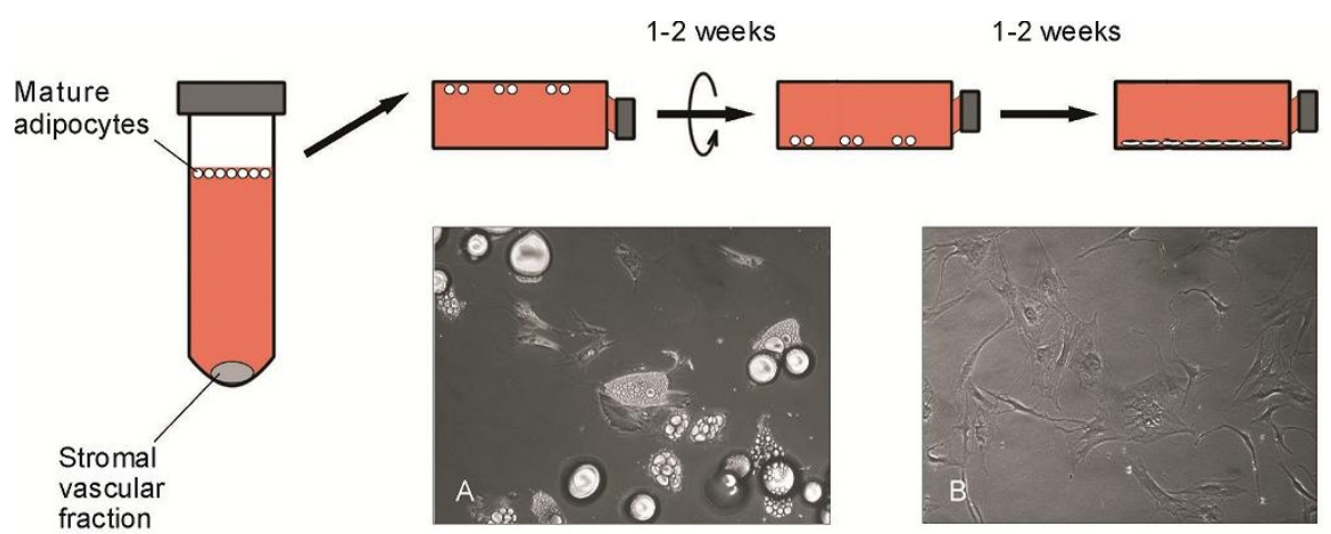

Figure 1 Establishment of the DFAT cells by modified ceiling culture method. (A) At the end of 1-2 weeks, most of the adhered adipocytes lost their unilocular outlook and presented multiple droplets. Then, lipid content decreased gradually in the following 1-2 weeks. (B) DFAT cells exhibited flat cell bodies, single nucleus and multiple processes. The size of DFAT cells correlated with their ancestor adipocytes based on the different amount of intracellular lipid amount. Bar: $100 \mu \mathrm{m}$.

Distinct to the ASCs, DFAT cells derived from ceiling culture method were identified as a more homogenous cell group in most reports [16-19, 23-26]. Although, this simple technique needs no complex procedures such as transfection or chemical induction, several key points should still be paid attention to. First, a thorough digestion and disperse is required to obtain purified adipocytes. Fat tissue has complex cellular composition, including adipocytes, ASCs, preadipocyte, fibroblasts, blood cells, endothelial cells, pericytes, smooth muscle cells and so on $[3,9,25,27-32]$. The purity of derived DFAT cells might be compromised by the contamination of those 
cells above when they're attached to the floating adipocytes after incomplete enzymatic treatment. To obtain uniform adipocyte suspension, it is recommended to perform adequate trituration and repeat filtration, wash and centrifuge. Immunofluorescence staining and/or flow cytometry prior to ceiling step will also help to verify the percentage of adipocytes within the isolated cells [25]. Secondly, at the beginning stage of the attachment between the adipocytes and the flask roof, minimal interfering is crucial to the transferring to the DFAT cells. Detached adipocytes showed very low percentage that undergo with the dedifferentiation process.

The mechanism under the dedifferentiation of mature adipocytes has not been clarified. In mammals, terminally differentiated cells largely lose the ability of reversing the differentiation process [14, 33-34]. However, recently studies demonstrated the opposite evidence that dedifferentiation could occur in defined situations on many cell types. Mouse myotubes can dedifferentiate and enter the cell cycle when stimulated with an extract prepared from newt regenerating limb tissue [35]. Monolayer culture of adult human cartilage chondrocytes invariably leads to their dedifferentiation in which cells regain the proliferation and multipotent abilities [23]. Furthermore, dedifferentiation also happens during some rare pathological occasions, which has been found in skeletal neoplasms such as osteosarcoma [36] and chondrosarcoma [37]. Kusafuka [38] recently reported a case of dedifferentiated epithelial-myoepithelial carcinoma (EMC) in the parotid region, based on the dedifferentiation of vascular smooth muscle cells. For the adipose tissue, a 20-year survey on the soft tissue sarcoma reported that 5 cases out of 5333 have been identified as dedifferentiated liposarcoma, while the overall number of liposarcoma was 652 [39]. Despite the malignant outcome, in the free-fat transfer in plastic and reconstructive surgery, some mature adipocytes also dedifferentiate into fibroblast-like cells under the ischemic conditions [21]. Hypoxia is also considered to be the main factor involved in not only the formation of DFAT cells in ceiling cultures, but also the dedifferentiation process of chondrocytes [40] and smooth muscle cells [41]. However, no evidence has been given that autologous or allogeneic implantation of DAFT cells into the human or animal bodies will lead to tumor or other diseases.

\section{Phenotype of DFAT cells}

DFAT cells have been constantly compared with the precursor cells in the SVF of adipose tissue, which can be divided into several categories as follows [25]: 1)
ASCs that can follow osteogenic, chondrogenic, adipogenic and other paths, 2) adipoblasts that have undergone determination to the adipose lineage, and 3) cells that have become committed to differentiate into preadipocytes. Most current studies on the DFAT cells have declared that they were a group of homogeneous cells. Similarities were also found on the immunophenotype compared with ASCs (Table 1), as well as other mesenchymal stem cells (MSCs) in cartilage and bone tissues. Stromal/stem cell-associated markers such as CD13, CD29, CD34, CD44 and CD90 were present on DFAT cells. The heterogeneous nature of ASCs exhibited on the surface antigen expressions mainly focused on the angiogenic markers such as CD31 and CD106, as well as certain MSCs related markers such as CD34 and CD49d. Another interesting characteristic of ASCs is that the surface immunophenotype partially changes in different passages. At the early passages (primary to 4th) of ASCs, the hematopoietic-associated markers (CD11a, CD14, CD45, CD86 and HLA-DR) decreased and the MSCs-associated markers (CD13, CD29, CD34, CD44, CD63, CD73, CD90 and CD166) increased significantly $[12,42]$. However, our unpublished work found that the phenotype of DFAT cells remained considerably stable from the early 2 nd to as long as the 30th passage. Both DFAT cells and ASCs had positive expression of HLA-A, $-B$ and $-C$, which indicated allogenic transplantation potential for both kinds.

A most recent microarray study on the gene expression patterns of certain surface antigens revealed that markers related to MSCs (CD44, CD140a, CD140b, CD146, CD266, CD325, and CD332), hematopoiesis (CD10, CD24, CD39, CD40, CD44, CD49e, CD56, CD71, CD86, CD87, CD109, CD138, CD225, and CD232) and myeloid lineage (CD10, CD13, CD40, CD44, CD49e, CD54, CD71, CD86, CD87, CD109, CD140a, and CD232) exhibited significant up-regulation during the dedifferentiation process from mature adipocytes toward DFAT cells. The same study also reported that during such process, there existed a significant decrease in functional phenotype-related genes and a parallel increase in cell proliferation, altered cell morphology, and regulation of the differentiation of related genes [45] In details, the down-regulation of genes involved in lipid metabolism such as adiponectin $(A D I P O Q)$, lipase, hormone sensitive (LIPE), pyruvate dehydrogenase kinase, isozyme 4 (PDK4), lipoprotein lipase $(L P L)$, fatty acid synthase $(F A S N)$, peroxisome proliferator-activated receptor c $(P P A R G)$, and fatty acid-binding protein 4 (FABP4) indicated that adipocyte phenotype diminished during the differentiation process of DFAT cells. On the other hand, a significant up-regulation of genes expre- 
ssion for cell movement, cell migration, tissue developmental processes, cell growth, cell proliferation, cell morphogenesis, altered cell shape, and cell differentiation had been found [45]. Those findings above were interpreted as that DFAT cells established multipotency during the dedifferentiation and such capacities demonstrated features of tissue-specific progenitor cells toward various lineages rather than earlier stage of stem cells.

Table 1 Comparison on the markers and surface antigens of DFAT cells and ASCs

\begin{tabular}{|c|c|c|}
\hline Markers & DFAT cells & ASCs \\
\hline Mac-1 (CD11b) & $-{ }^{\mathrm{a}}$ & $-{ }_{-b, c, d}$ \\
\hline Aminopeptidase N (CD13) & $+{ }^{a}, *$ & $++^{c, d}$ \\
\hline Integrin $\beta 1(\mathrm{CD} 29)$ & $+{ }^{\mathrm{a}, *}$ & $+{ }^{b, c, d}$ \\
\hline PECAM-1 (CD31) & $-{ }^{\mathrm{a}, *}$ & $+b /-c, d$ \\
\hline L-seletin ligand (CD34) & $-{ }^{\mathrm{a}, *}$ & $+{ }^{c} /-^{d}$ \\
\hline Pgp-1 (CD44) & $+{ }^{\mathrm{a}, *}$ & $+b, c, d$ \\
\hline LCA (CD45) & $-{ }^{\mathrm{a}}$ & $-{ }^{c, d}$ \\
\hline Integrin $\alpha 4$ (CD49d) & $+{ }^{a}$ & $+{ }^{b, d} /{ }^{c}$ \\
\hline NCAM isoform (CD56) & $-{ }^{\mathrm{a}}$ & $-{ }^{\mathrm{c}}$ \\
\hline Thy-1 (CD90) & $++^{\mathrm{a}}$ & $++^{b, d}$ \\
\hline Endoglin (CD105) & $+{ }^{\mathrm{a}, *}$ & $+{ }^{b, c, d}$ \\
\hline VCAM-1 (CD106) & $-{ }^{\mathrm{a}, *}$ & $+\mathrm{d} /-^{b, c}$ \\
\hline PDGFR $\beta$ (CD140b) & $-*$ & $+{ }^{b}$ \\
\hline MCAM (CD146) & $+*$ & $+c, d$ \\
\hline$\alpha$-SMA & $-*$ & $++^{d}$ \\
\hline HLA-A & $+{ }^{a}$ & $+{ }^{a}$ \\
\hline HLA-B & $+{ }^{a}$ & $++^{a}$ \\
\hline HLA-C & $+{ }^{\mathrm{a}}$ & $++^{\mathrm{a}}$ \\
\hline
\end{tabular}

+: positive expression; - : negative expression; *: results from our preliminary studies. a refers to [14]; b refers to [1]; c refers to [43]; d refers to [44].

\section{Multilineage differentiation ability}

Several independent studies have already reported that DFAT cells are capable of differentiation into multiple mesenchymal lineages, including osteogenic, adipogenic, chondrogenic and myogenic abilities. However, multipotency of MSCs was usually tested by a series of differentiation assays in vitro, so were the DFAT cells. In vitro positive staining of alizarin red/von Kossa (osteogenesis), oil red $\mathrm{O}$ (adipogenesis) and alcian blue (chondrogenesis) with nonclonal cell cultures would have a compromised base for their conclusions. Therefore, those assays on DFAT cells with clonal analysis could provide us with more reliable data. In the first comprehensive differentiation ability test on DFAT cells with such measures carried out by Mugishima, it was found that clonally derived DFAT cells were multipotent cells, and they were heterogeneous in terms of their differentiation potential. $25 \%$ of the clones had triple lineage ability, while the double and single lineage clones share the remaining portion with each a percentage of $37.5 \%$ [14]. This mesenchymal nature resembled the previous findings on ASCs and other MSCs in bone, muscle and cartilage tissues. However, ACSs have been verified to have a broader potential of differentiation not only into mesenchymal lineages, but also toward endothelial cells, epithelial cells, hepatocytes and neurons [46]. Although it was pointed out that outcomes of the experiments in vivo with the same cell strains have been reviewed to have a relatively weak correlation with the result got in vitro [47], most of the following cell culture and animal experiments on the differentiation abilities of DFAT cells shared common results.

\section{Adipogenesis}

Redifferentiation of DFAT cells toward adipocytes was not spontaneous. To our study, DFAT cells remained stable and strong proliferation ability after 30th passage without detectable establishment of lipid droplet inside plasma, which was consistent with other reports [24]. PCR analysis showed that although DFAT cells had decreased level of LPL, leptin and glucose transporter 4 (GLUT4) compared with mature adipocytes, they still express key adipogenic markers including peroxisome proliferator-activated receptor gamma (PPAR $\gamma$ ), CAAT/ enhancer-binding proteins $(\mathrm{C} / \mathrm{EBP} \alpha, \mathrm{C} / \mathrm{EBP} \beta$ and $\mathrm{C} / \mathrm{EBP} \delta$ ) and sterol regulatory element-binding protein-1c (SREBP1c) $[14,24-25]$. In vitro, several recipes of adipogenic cultures were applied with different combination and dosage of induction chemicals such as dexamethasone, 3-isobutyl-1-methylxanthine and insulin transferring selenium X (ITS) [14-15, 18]. Initial establishment of lipid droplets could found as early as 5-7 days of induction, while significant amount of lipid accumulation within cells could be observed after 2-3 weeks. In vivo experiment showed same adipogenic capacity of DFAT cells. Direct injection of DFAT cell suspension by syringes into the subcutaneous portion over sternum of mice resulted in fat pad formation free of chemical induction after 3 weeks [24-25].

\section{Osteogenesis and Chondrogenesis}

Expression of Runx2, osteopntin, osterix, osteocalcin, PTHrl and SOX9 indicated the osteogenic and chondrogenic potentials of DFAT cells, respectively [14]. Differentiation toward osteoblasts was commonly performed by adding dexamethasone, $\beta$-glycerophosphate and L-ascorbic acid-2-phosphate into the culture medium in vitro. It should be noticed that both isoforms of 
$\operatorname{PPAR} \gamma, \operatorname{PPAR} \gamma 1$ and PPAR $\gamma 2$, could be stimulated by dexamethasone. Therefore, the amount of dexamethasone in osteogenic induction is usually significantly lower than adipogenic induction. Osteogenic differentiation of DFAT cells could also be induced by all-trans retinoic acid, an analog of retinol which interacts with bone morphogenetic proteins (BMPs) to inhibit adipogenesis and enhance osteogenesis [18]. Chondrogenic induction could be facilitated with L-ascorbic acid-2-phosphate, proline, pyruvate, transforming growth factor $\beta 3$ (TGF$\beta 3$ ) and ITS. After 3-4 weeks of induction, calcified matrix deposition was verified by alkaline phosphate (ALP), alizarin red S and von Kossa staining and chondrocyte transformation was observed with alcian blue staining [14]. Implantation of DFAT cells along with collagen-based scaffolds also demonstrated their differentiation abilities into osteoblasts and chondrocytes in vivo $[14,18]$.

\section{Myogenesis}

Myocytes can be divided into three categories: skeletal, cardiac and smooth muscle cells, which present various cellular characteristics and biological behaviors. Myogenesis is the formation of muscular cells and tissue, a multiple-stage process composed of the proliferation of myoblasts, secretion of fibronectin onto their extracellular matrix, alignment of the myoblasts into multi-nucleated myotubes and cell fusions [48]. Two muscle-specific transcription factors, Myf5 and $\mathrm{MyoD}$, are actively involved in the regulation of such process. MyoD was identified in DFAT cells with a high degree of methylation in its regulatory region. Treatment of 5-azacytidine (Aza-C), a demethylating agent, led to the expression of MyoD and myogenin in DFAT cells, as well as the formation of multinucleated cells expressing myosin heavy chain (MHC), although Myf5 was still absent after induction [19]. Another study found that DFAT cells converted into cardiomyocytes in vitro after culture with native cardiomyocytes or stem cell methylcellulose medium. In the same study, injection of DFAT cells into the rat ischemic heart model induced neovascularization and cardiac tissue rehabilitation [16]. Results above suggested that under proper conditions, DFAT cells could undertake myogenic differentiation both in vivo and in vitro.

\section{Angiogenesis}

One distinct aspect of the differentiation capacities between DFAT cells and ASCs existed in their angiogenesis behaviors. Blood vessels are composed of an interior surface of endothelial cell and perivascular supporting parts of smooth muscle cells and pericytes. A subset of CD34 positive hematopoietic stem cells could differentiate to the endothelial lineage and express endothelial markers and incorporated 1,1'-dioetadeeyl3,3,3',3'-tetramethylindocarboeyanine labeled acetylated low density lipoprotein (Dil-ac-LDL) [49]. Similar characteristics could be found on ASC in vitro and in vivo when angiogenic agents such as fibroblast growth factors (FGF), epidermal growth factor (EGF) and vascular endothelial growth factor VEGF were applied [50-53]. Intro experiments also revealed that DFAT cells could elicit neovascularization and promote angiogenesis $[16$, 54]. However, no report has been published on induction of DFAT cells toward endothelial lineage in vitro. Meanwhile, our preliminary experiment and other reports all found that DFAT cells with or without induction have negative expression of endothelial cell or progenitor markers such as such von Willebrand factor (vWF), CD31 and CD34 [14]. Interestingly, DFAT cells cultured on the Matrigel sprouted and joined into vascular networks, which was identical to the structure formed by endothelial cells. We interpreted this phenomenon as the perivascular nature of DFAT cells. Our studies results supported this explanation by key pericyte-related markers found on DFAT cells such as CD140b and NG2.

Comparison on the multipotent abilities between DFAT cells and ASCs should be resulted from a cross check and review on previous literatures. Although current reports on ASCs significantly outnumber those on DFAT cells, the similarity on the mesenchymal stem cell capacity could be found on both lineages. Our novel findings above on the perivascular behavior of DFAT cells were consistent to the new understandings of MSCs. Recent studies identified that pericytes in multiple organs and tissues could demonstrate perivascular markers (CD146, NG2 and CD140b) and MSCs markers (CD44, CD73, CD90, CD 105), exhibiting osteogenic, chondrogenic and adipogenic potentials [55-57]. MSCs within adult mesenchymal tissues such as bone and adipose also could differentiate into or act as pericytes without induction of growth factors [58-59]. Therefore, blood vessel walls were considered as a source of progenitor cells, which gave a common origin of MSCs and other tissue-specific adult stem cells [60]. Both MSCs and pericyte characteristics of DFAT cells might be derived from the association and interaction of mature adipocytes and micro vascular networks within the adipose tissue.

\section{Summary}

Mature adipocytes have been considered as the terminally differentiated lineage without proliferation ability. However, simple ceiling culture technique using their 
buoyancy gave rise to the plasticity of adipocytes. The newly established DFAT cells exhibited vigorous proliferation and multipotent abilities with significant advantages over other adult stem cells. Compared with bone-marrow derived stem cells, most white adipose tissues locate in subcutaneous positions and their abundance is usually guaranteed. Harvest of mature adipocytes requires less invasive method such as commonly used liposuction by a fine needle, which pose much less physical and psychological impact to the donor. Compared with ASCs, DFAT cells represent a more homogeneous nature. Modified ceiling culture method reduces the chance of contamination by other cell source in SVF portion to the minimal. Additionally, the expansion capacity and the phenotype of DFAT cells are also more stable, without shift of cellular markers or spontaneous redifferentiation after dozens of passages. In vitro and/or in vivo experiments have revealed the adipogenic, osteogenic, chondrogenic and myogenic potentials of DFAT cells. Although no human trail on such cells has ever been reported, it could not hinder us from looking forward to their clinical application, especially in oral and maxillofacial regeneration. One of the ongoing studies in our group tries to derive DFAT cells from the human buccal fat pad removed in facial cosmetic surgeries. Objectives of following experiments involve the further possible autologous or allogeneic transplanttation. Other previous in vivo studies on the osteogenic, chondrogenic and myogenic capacities of DFAT cells provided alternative treatment thoughts for bone and cartilage loss, skeletal muscle shrinkage and ischemic heart disease. Furthermore, perivascular characteristics of those cells not only support the novel understanding of MSCs, but also showed potential usage in vascular reconstruction and repair in large tissue damages. However, it must be pointed out the cellular nature of DFAT cells still remains unclarified, neither did their differentiation stage. Considering the occurrence of dedifferentiated liposarcoma in nature, the biological safety of their implantation requires more long term animal or preclinical studies.

\section{References}

1 Katz AJ, Tholpady A, Tholpady SS, Shang H, Ogle RC. Cell surface and transcriptional characterization of human adipose-derived adherent stromal (hADAS) cells. Stem Cells 2005; 23: 412-423.

2 Rigotti G, Marchi A, Sbarbati A. Adipose-derived mesenchymal stem cells: past, present, and future. Aesthetic Plast Surg 2009; 33: 271-273.

3 Gimble J, Guilak F. Adipose-derived adult stem cells: iso- lation, characterization, and differentiation potential. Cytotherapy 2003; 5: 362-369.

4 Locke M, Windsor J, Dunbar PR. Human adipose-derived stem cells: isolation, characterization and applications in surgery. ANZ J Surg 2009; 79: 235-244.

5 Bunnell B, Flaat M, Gagliardi C, Patel B, Ripoll C. Adipose-derived stem cells: Isolation, expansion and differentiation. Methods 2008; 45: 115-120.

6 Gimble JM, Katz AJ, Bunnell BA. Adipose-derived stem cells for regenerative medicine. Circ Res 2007; 100: 1249 1260 .

7 Dudas JR, Losee JE, Penascino VM, et al. Leporine-derived adipose precursor cells exhibit in vitro osteogenic potential. J Craniofac Surg 2008; 19: 360-368.

8 Taniguchi M, Guan L, Zhang B, et al. Adipogenesis of bovine perimuscular preadipocytes. Biochem Biophy Res Commun 2008; 366: 54-59.

9 Griesche N, Luttmann W, Luttmann A, et al. A simple modification of the separation method reduces heterogeneity of adipose-derived stem cells. Cells Tissues Organs 2010; 192: 106-115.

10 Liu J, Huang J, Lin T, Zhang C, Yin X. Cell-to-cell contact induces human adipose tissue-derived stromal cells to differentiate into urothelium-like cells in vitro. Biochem Biophys Res Commun 2009; 390: 931-936.

11 Conde-Green A, Baptista LS, de Amorin NF, et al. Effects of centrifugation on cell composition and viability of aspirated adipose tissue processed for transplantation. Aesthet Surg $J$ 2010; 30: 249-255.

12 Mitchell JB, McIntosh $\mathrm{K}$, Zvonic $\mathrm{S}$, et al. Immunophenotype of human adipose-derived cells: temporal changes in stromal-associated and stem cell-associated markers. Stem Cells 2006; 24:376-385.

13 Sugihara H, Yonemitsu N, Miyabara S, Yun K. Primary cultures of unilocular fat cells: characteristics of growth in vitro and changes in differentiation properties. Differentiation 1986; 31: 42-49.

14 Matsumoto T, Kano K, Kondo D, et al. Mature adipocytederived dedifferentiated fat cells exhibit multilineage potential. J Cell Physiol 2008; 215: 210-222.

15 Zhang HH, Kumar S, Barnett AH, Eggo MC. Ceiling culture of mature human adipocytes: use in studies of adipocyte functions. J Endocrinol 2000; 164: 119-128.

16 Jumabay M, Matsumoto T, Yokoyama S, et al. Dedifferentiated fat cells convert to cardiomyocyte phenotype and repair infarcted cardiac tissue in rats. $\mathrm{J} \mathrm{Mol} \mathrm{Cell} \mathrm{Cardiol}$ 2009; 47: 565-575.

17 Hildner F, Concaro S, Peterbauer A, et al. Human adipose-derived stem cells contribute to chondrogenesis in coculture with human articular chondrocytes. Tissue Eng Part A 2009; 15: 3961-3969.

18 Oki Y, Watanabe S, Endo T, Kano K. Mature adipocyte- 
derived dedifferentiated fat cells can trans-differentiate into osteoblasts in vitro and in vivo only by all-trans retinoic acid. Cell Struct Funct 2008; 33: 211-222.

19 Kazama T, Fujie M, Endo T, Kano K. Mature adipocytederived dedifferentiated fat cells can transdifferentiate into skeletal myocytes in vitro. Biochem Biophys Res Commun 2008; 377: 780-785.

20 Shigematsu M, Watanabe H, Sugihara H. Proliferation and differentiation of unilocular fat cells in the bone marrow. Cell Struct Funct 1999; 24: 89-100.

21 Tholpady SS, Aojanepong C, Llull R, et al. The cellular plasticity of human adipocytes. Ann Plast Surg 2005; 54: 651-656.

22 Coradeghini R, Guida C, Scanarotti C, et al. A comparative study of proliferation and hepatic differentiation of human adipose-derived stem cells. Cells Tissues Organs 2010; 191: 466-477.

23 de la Fuente Rdl, Abad JL, García-Castro J, et al. Dedifferentiated adult articular chondrocytes - a population of human multipotent primitive cells. Exp Cell Res 2004; 297: 16.

24 Yagi K, Kondo D, Okazaki Y, Kano K. A novel preadipocyte cell line established from mouse adult mature adipocytes. Biochem Biophys Res Commun 2004; 321: 967-974.

25 Nobusue H, Endo T, Kano K. Establishment of a preadipocyte cell line derived from mature adipocytes of GFP transgenic mice and formation of adipose tissue. Cell Tissue Res 2008; 332: 435-446.

26 Matsumoto T, Kano K, Kondo D, et al. Mature adipocytederived dedifferentiated fat cells exhibit multilineage potential. J Cell Physiol 2008; 215: 210-222.

27 Traktuev DO, Merfeld-Clauss S, Li J, et al. A population of multipotent CD34-positive adipose stromal cells share pericyte and mesenchymal surface markers, reside in a periendothelial location, and stabilize endothelial networks. Circ Res 2008; 102: 77-85.

28 Rodriguez AM, Elabd C, Delteil F, et al. Adipocyte differentiation of multipotent cells established from human adipose tissue. Biochem Biophys Res Commun 2004; 315: 255-263.

29 Poglio S, De Toni-Costes F, Arnaud E, et al. Adipose tissue as a dedicated reservoir of functional mast cell progenitors. Stem Cells 2010; 28: 2065-2072.

30 Hong L, Peptan IA, Colpan A, Daw JL. Adipose tissue engineering by human adipose-derived stromal cells. Cells Tissues Organs 2006; 183: 133-140.

31 Madonna R, De Caterina R. Adipose tissue: a new source for cardiovascular repair. J Cardiovasc Med (Hagerstown) 2010; 11: 71-80.

32 Lin Y, Chen X, Yan Z, et al. Multilineage differentiation of adipose-derived stromal cells from GFP transgenic mice. Mol Cell Biochem 2006; 285: 69-78.
33 Walsh K, Perlman H. Cell cycle exit upon myogenic differentiation. Curr Opin Genet Dev 1997; 7: 597-602.

34 Andres V, Walsh K. Myogenin expression, cell cycle withdrawal, and phenotypic differentiation are temporally separable events that precede cell fusion upon myogenesis. $J$ Cell Biol 1996; 132: 657-666.

35 McGann CJ, Odelberg SJ, Keating MT. Mammalian myotube dedifferentiation induced by newt regeneration extract. Proc Natl Acad Sci U S A 2001; 98: 13699-13704.

36 Yoshida A, Ushiku T, Motoi T, et al. Well-differentiated liposarcoma with low-grade osteosarcomatous component: an underrecognized variant. Am J Surg Pathol 2010; 34: 1361-1366.

37 Kumta SM, Griffith JF, Chow LT, Leung PC. Primary juxtacortical chondrosarcoma dedifferentiating after 20 years. Skeletal Radiol 1998; 27: 569-573.

38 Kusafuka K, Takizawa Y, Ueno T, et al. Dedifferentiated epithelial-myoepithelial carcinoma of the parotid gland: a rare case report of immunohistochemical analysis and review of the literature. Oral Surg Oral Med Oral Pathol Oral Radiol Endod 2008; 106: 85-91.

39 Wibmer C, Leithner A, Zielonke N, Sperl M, Windhager R. Increasing incidence rates of soft tissue sarcomas? A population-based epidemiologic study and literature review. Ann Oncol 2010; 21: 1106-1111.

40 Lafont JE. Lack of oxygen in articular cartilage: consequences for chondrocyte biology. Int J Exp Pathol 2010; 91 : 99-106.

41 Aitken KJ, Tolg C, Panchal T, et al. Mammalian target of rapamycin (mTOR) induces proliferation and de-differentiation responses to three coordinate pathophysiologic stimuli (mechanical strain, hypoxia, and extracellular matrix remodeling) in rat bladder smooth muscle. Am J Pathol 2010; 176: 304-319.

42 McIntosh K, Zvonic S, Garrett S, et al. The immunogenicity of human adipose-derived cells: temporal changes in vitro. Stem Cells 2006; 24: 1246-1253.

43 Gronthos S, Franklin DM, Leddy HA, et al. Surface protein characterization of human adipose tissue-derived stromal cells. J Cell Physiol 2001; 189: 10.

44 Schaffler A, Buchler C. Concise review: adipose tissuederived stromal cells - basic and clinical implications for novel cell-based therapies. Stem Cells 2007; 25: 818-827.

45 Ono H, Oki Y, Bono H, Kano K. Gene expression profiling in multipotent DFAT cells derived from mature adipocytes. Biochem Biophys Res Commun 2011; 407: 562-567.

46 Franco Lambert AP, Fraga Zandonai A, Bonatto D, Cantarelli Machado D, Pêgas Henriques JA. Differentiation of human adipose-derived adult stem cells into neuronal tissue: does it work? Differentiation 2009; 77: 221-228.

47 Bianco P, Kuznetsov SA, Riminucci M, Gehron Robey P. Postnatal skeletal stem cells. Methods Enzymol 2006; 419: 
$117-148$.

48 Vlahopoulos S, Zimmer WE, Jenster G, et al. Recruitment of the androgen receptor via serum response factor facilitates expression of a myogenic gene. $J$ Biol Chem 2005; 280: 7786-7792.

49 Urbich C, Dimmeler S. Cell biology of Leydig cells in the testis. Circ Res 2004; 95: 343-353.

50 Gehling UM, Ergu" $\mathrm{n}$ Sl, Schumacher U, et al. In vitro differentiation of endothelial cells from AC133-positive progenitor cells. Blood 2000; 95: 17.

51 Hebert TL, Wu X, Yu G, et al. Culture effects of epidermal growth factor (EGF) and basic fibroblast growth factor (bFGF) on cryopreserved human adipose-derived stromal/ stem cell proliferation and adipogenesis. $J$ Tissue Eng Regen Med 2009; 3: 553-561.

52 Froehlich H, Gulati R, Boilson B, et al. Carotid repair using autologous adipose-derived endothelial cells. Stroke 2009; 40: $1886-1891$.

53 Miranville A. Improvement of postnatal neovascularization by human adipose tissue-derived stem cells. Circulation 2004; 110: 349-355.

54 Casteilla L, Planat-Benard V, Cousin B, et al. Plasticity of human adipose lineage cells toward endothelial cells: physi- ological and therapeutic perspectives. Circulation 2004; 109: 656-663.

55 He W, Nieponice A, Soletti L, et al. Pericyte-based human tissue engineered vascular grafts. Biomaterials 2010; 31: $8235-8244$.

56 Chen C-W, Montelatici E, Crisan M, et al. Perivascular multi-lineage progenitor cells in human organs: regenerative units, cytokine sources or both? Cytokine Growth Factor Rev 2009; 20:429-434.

57 Farrington-Rock C. Chondrogenic and adipogenic potential of microvascular pericytes. Circulation 2004; 110: 2226 2232.

58 Natesan S, Zhang G, Baer DG, et al. A bilayer construct controls adipose-derived stem cell differentiation into endothelial cells and pericytes without growth factor stimulation. Tissue Eng Part A 2011; 17: 941-953.

59 Bexell D, Gunnarsson S, Tormin A, et al. Bone marrow multipotent mesenchymal stroma cells act as pericyte-like migratory vehicles in experimental gliomas. Mol Ther 2008; 17: 183-190.

60 Crisan M, Yap S, Casteilla L, et al. A Perivascular origin for mesenchymal stem cells in multiple human organs. Cell Stem Cell 2008; 3 : 301-313. 